Gut, 1987, 28, S1, 159-164

\title{
Adaptation of the jejunal mucosa in the experimental blind loop syndrome: changes in paracellular conductance and tight junction structure
}

\author{
J-D SCHULZKE, M FROMM, C J BENTZEL, H MENGE, AND E-O RIECKEN \\ From the Abteilung für Gastroenterologie, Klinikum Steglitz, Freie Universität Berlin, FRG, Institut für \\ Klinische Physiologie, Klinikum Steglitz, Freie Universität Berlin, FRG, and Department of Medicine, East \\ Carolina University, Greenville, North Carolina, USA.
}

SUMMARY Self-filling blind loops of rat jejunum exhibit hyperregenerative transformation of the mucosa. We used this experimental model to characterise mechanisms, which may occur under similar conditions in man (stagnant loop syndrome). Epithelial and subepithelial resistance were measured in the Ussing-chamber by voltage divider ratio measurements after positioning a microelectrode between epithelium and subepithelial tissue layers. In the blind loop, epithelial resistance increased from $8 \pm 1$ to $23 \pm 1 \Omega \mathrm{cm}^{2}$ and subepithelial resistance from $39 \pm 4$ to $86 \pm 8 \Omega \mathrm{cm}^{2}$ as compared with control jejunum. The increase in the subepithelial resistance was paralleled anatomically by an increase in the thickness of the subepithelial tissue layers from $63 \pm 4 \mu \mathrm{m}$ to $177 \pm 19 \mu \mathrm{m}$. Ultrastructural analysis of the tight junction area by freeze fracture electron microscopy revealed an increase in the total junctional 'depth' in the crypts from $243 \pm 9 \mathrm{~nm}$ in control jejunum to $396 \pm 17 \mathrm{~nm}$ in the blind loop, while the number of horizontally oriented 'strands' remained unchanged. Villus tight junctions did not differ between blind loop and control. We interpret the alterations in the self-filling blind loop as an adaptive response of the epithelium which reduces backleakage of already absorbed electrolytes across the tight junction into the intestinal lumen. This mechanism is suitable to support the intestine in maintaining body electrolyte and water contents during cellular electrolyte malabsorption.

The morphological adaptive response of the intestinal mucosa to various types of stress is restricted to three different patterns: hypoplasia, hyperplasia and hyperregenerative transformation,,$^{1,2}$ the last of which can be experimentally provoked in the self-filling blind loop in the rat. This adaptive pattern is characterised by increased crypt length with enhanced cell proliferation and alterations in villus height which vary in length depending on the intensity of luminal noxious factors. $^{3}$ In the blind loop syndrome, stasis of the ingesta leads to bacterial overgrowth which is accompanied by bacterial deconjugation of bile acids. Three factors, the stasis per se, bacterial proteases and unphysiologically high concentrations of free bile acids have been shown to be involved in the observed transformation of the mucosa ${ }^{3}$ and are also relevant in man in the stagnant loop syndrome. ${ }^{4}$ Thus, the

Address for correspondence: Dr J-D Schulzke, Medizinische Klinik, Klinikum Steglitz, Hindenburgdamm 30, 1000 Berlin 45, FRG. experimental self-filling blind loop in the rat may be a useful model to study principal mechanisms of intestinal structural and functional adaptation in this condition.

In order to guarantee electrolyte and water absorption, intestinal cells are equipped with tight junctions to barricade the paracellular space from the lumen. Thus, beside active cellular transport systems the tight junction determines the ability of an epithelium to perform active net absorption. Suitable techniques for characterisation of the tight junction include both ultrastructural measurements on freeze fracture replicas of the tight junction as well as epithelial resistance measurements in the small intestine, since in leaky epithelia the electrical resistance of the epithelial cell layer is mainly determined by the paracellular pathway. ${ }^{5}$ Using these techniques in the gall bladder of Necturus maculosus, parallel changes in epithelial resistance and tight junctional structure 
have already been demonstrated and these were accompanied by an increase in net water absorption. ${ }^{6}$

The aim of this study was to define the tight junction of the hyper-regeneratively transformed intestinal mucosa in this respect.

\section{Methods}

\section{ANIMALS}

Male albino Wistar rats (180-200 g) were kept on a standard diet and tap water ad libitum. Jejunal selffilling blind loops were surgically created $12 \mathrm{~cm}$ distal to the ligamentum of Treitz as described earlier. ${ }^{3}$ Four weeks later, self-filling blind loops and corresponding segments of control jejunum were excised, opened along the mesenteric border, rinsed free from ingesta and mounted into Ussing-chambers. The perfusion medium consisted of (in $\mathrm{mM}$ ): $\mathrm{Na} \mathrm{140,}$ $\mathrm{K} 5 \cdot 4, \mathrm{Mg} \mathrm{1} \cdot 2, \mathrm{Ca} \mathrm{1} \cdot 2, \mathrm{Cl} 123 \cdot 8, \mathrm{HCO}_{3} 21, \mathrm{HPO}_{4} 2 \cdot 4$, $\mathrm{H}_{2} \mathrm{PO}_{4} 0.6$. The solution was gassed with a mixture of $95 \% \mathrm{O}_{2}$ and $5 \% \mathrm{CO}_{2}$ and had a pH of 7.4 at $37^{\circ} \mathrm{C}$.

Voltage divider ratio measurements were performed as described earlier. ${ }^{7}$ Briefly, bipolar DC current pulses $\left(200 \mathrm{~ms} ; 0.1 \mathrm{~Hz} ; \pm 50 \mu \mathrm{A} / \mathrm{cm}^{2}\right)$ were passed through the tissue for measuring total tissue resistance. The fractions of epithelial and subepithelial resistance were then obtained by positioning the tip of a microelectrode between these two structures and measuring the voltage divider ratio. For this purpose the microelectrode was manipulated by a piezo stepper. ${ }^{8}$ Criteria for successful impalements were: (i) abrupt voltage deflections while entering an epithelial cell through the apical membrane and while just penetrating the basolateral membrane; (ii) similar deflections immediately when retracting the electrode.

Freeze fracture electron microscopy was carried out as described earlier. ${ }^{\circ}$ Shortly, the tissues were fixed in the Ussing-chamber with $2 \%$ phosphate buffered glutaraldehyde, frozen in Freon and liquid nitrogen $\left(-100^{\circ} \mathrm{C}\right)$, and shadowed with platinum and carbon in a Denton DFE-3 apparatus. Freeze fracture replicas were scanned using a Siemens 1A electron microscope. Morphometric analysis was carried out on coded prints (total magnification 60000 ) using all tight junctional regions where both a luminal and an abluminal strand of the meshwork could be seen. For morphometrical analysis, grid lines were drawn on electronmicrograph prints perpendicular to the most luminal strand of the junction at $167 \mathrm{~nm}$ intervals (equivalent to $1 \mathrm{~cm}$ on the print). The number of intersections between grid lines and strands were counted and defined as the number of more or less horizontally oriented strands ('strands') within the total junction. The vertical expansion of the tight junction ('depth') is the distance between the most luminal and contraluminal strand. The thickness of the subepithelial tissue ('d') was defined in cross sections (HE-stained) as the distance between the lower crypt endings and the serosal surface (Fig. 1).

All values given are means \pm SEM. Statistical analysis is based on Student's $t$ test.

\section{Results}

Under in vivo conditions, intestinal transport is only influenced by the resistance of the epithelial cell layer, since capillaries are located in close distance to epithelial cells. Therefore, we applied a technique to determine the epithelial resistance selectively (Table 1). It turned out that epithelial resistance increased three fold in the blind loop as compared to control jejunum. Concomitantly, there was a two fold increase in subepithelial resistance, which correlates with an increase in the thickness of the subepithelial tissue layers in the blind loop (see below).

Values for epithelial and subepithelial resistance in the blind loop and in control jejunum obtained by voltage divider ratio measurements were not significantly different from according values (unpublished results) obtained by another established method, namely AC-impedance analysis. ${ }^{6}$ ?

Because in leaky epithelia the epithelial resistance is

Table 1 Epithelial and subepithelial resistance of selffilling blind loops and corresponding control jejunum.

\begin{tabular}{lccc}
\hline & $\begin{array}{l}\text { Epithelial } \\
\text { resistance }\end{array}$ & $\begin{array}{l}\text { Subepithelial } \\
\text { resistance }\end{array}$ & $n$ \\
\hline control & $8 \pm 1$ & $39 \pm 4$ & 6 \\
blind loop & $23 \pm 1$ & $86 \pm 8$ & 6 \\
P & $<0.001$ & $<0.001$ & \\
\hline
\end{tabular}

Values are means \pm SEM. Results were obtained from voltage divider ratio measurements (see Methods). All resistance values refer to the serosal surface area.

Table 2 Tight junction structure of self-filling blind loops and corresponding control jejunum

\begin{tabular}{lccc}
\hline & Strands & Depth $(\mathrm{nm})$ & $n$ \\
\hline Villus region: & & & \\
$\quad$ control & $5 \cdot 48 \pm 0 \cdot 11$ & $215 \pm 5$ & 143 \\
blind loop & $5 \cdot 37 \pm 0 \cdot 12$ & $231 \pm 7$ & 86 \\
P & $\mathrm{NS}$ & $\mathrm{NS}$ & \\
Crypt region: & & & \\
$\quad$ control & $5 \cdot 81 \pm 0 \cdot 16$ & $243 \pm 9$ & 132 \\
blind loop & $5 \cdot 76 \pm 0.08$ & $396 \pm 17$ & 412 \\
P & $\mathrm{NS}$ & $<0.001$ & \\
\hline
\end{tabular}

Tight junction structure in the villus and crypt region. Strands: number of horizontally oriented strands in the tight junction (see Methods). Depth: vertical expansion of the total tight junction in $\mathrm{nm}$ measured on reprints (at 60000 fold magnification). 

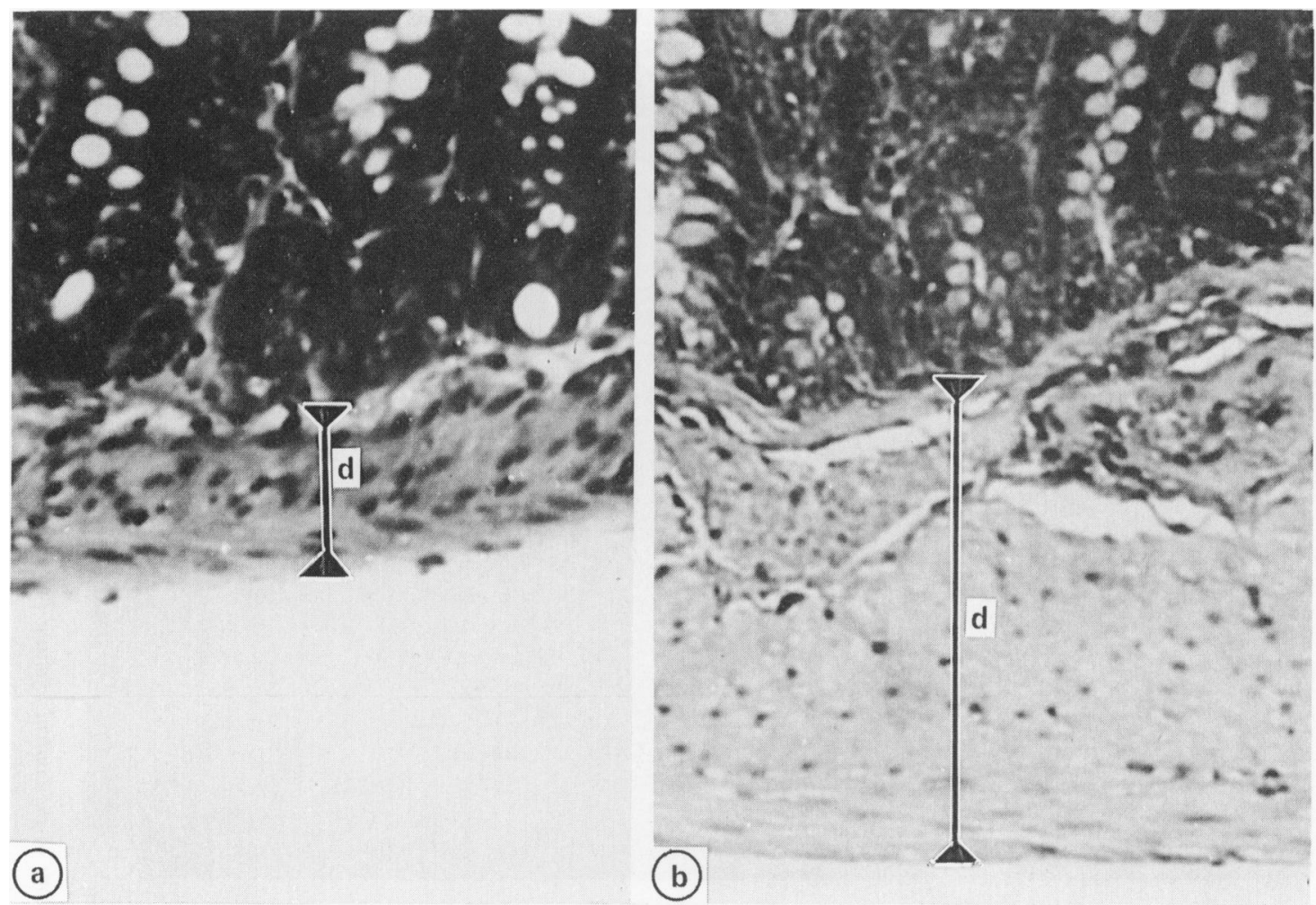

Fig. 1 Typical cross section obtained by conventional histology (HE-stained) of the control jejunum (a) and the self-filling blind loop $(b)$. ' $d$ ': thickness of the subepithelial tissue layers.

mainly determined by the paracellular pathway and there mainly by the tight junctional border, ${ }^{10}$ we analysed the tight junction structure. In Figure 2, typical freeze fracture replicas from crypt cell tight junctions are presented for the control jejunum and the blind loop. In the blind loop the 'depth' of the tight junction is increased. The results of the morphometrical analysis are compiled in Table 2 . Crypt tight junctions showed an increased 'depth' (number of 'strands' were unchanged) when compared with tight junctions of the villi (this applies for the control jejunum and the blind loop). Under the blind loop condition, crypt tight junction 'depth' increased as compared to control while the number of 'strands' remained unaltered. No effect on the tight junction structure of the villi could be seen (neither on 'depth' nor on 'strand' number) indicating that the observed change of epithelial resistance was not attributed to this region.

Furthermore, we compared the thickness of the subepithelial tissue layers ('d') (Fig. 1). In the blind loop, ' $d$ ' $(177 \pm 19 \mu \mathrm{m} ; \mathrm{n}=8)$ was higher than in the controls $(63 \pm 4 \mu \mathrm{m} ; \mathrm{n}=8)$. Thus, the increase in subepithelial resistance was accompanied by an increase in the thickness of the subepithelial tissue layers. A complete numerical agreement, however, was not expected, as the connective tissue within the villi and in between the crypts (the main part of the lamina propria mucosae) was not included in parameter ' $\mathrm{d}$ ' but contributes to the subepithelial resistance.

\section{Discussion}

In the experimental blind loop syndrome, the mucosa in the self-filling blind loop is hyperregeneratively transformed. This study aimed at the hitherto unknown role of the tight junction area in electrolyte and water transport under this condition. Using combined electrical and morphological techniques three relevant findings have been obtained: (i) The absolute value of the epithelial resistance in the normal jejunum - measured for the first time - was found to be surprisingly low. (ii) Epithelial and subepithelial resistance in the blind loop were increased considerably. (iii) The functional changes 

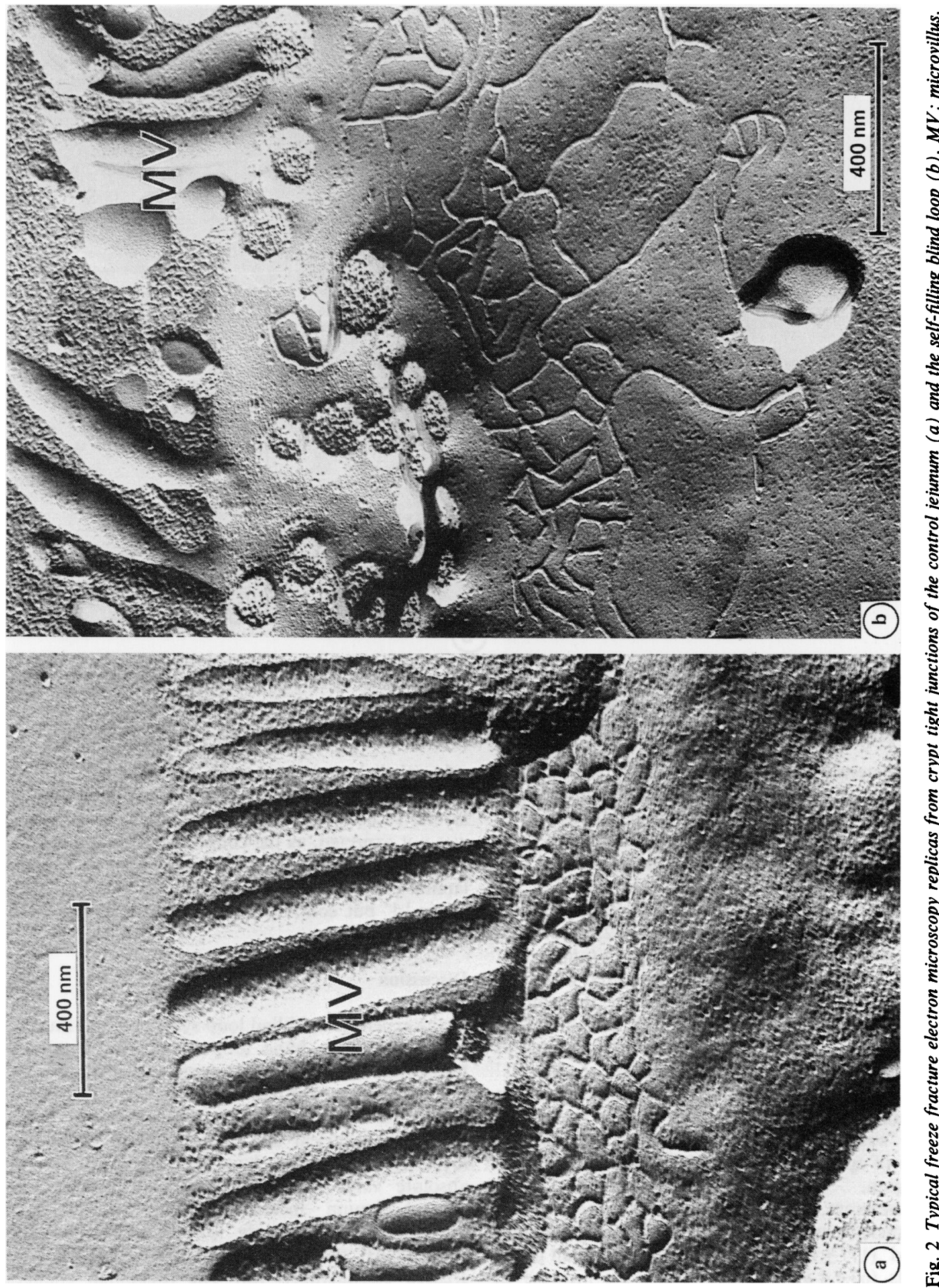
were paralleled by an increase in depth of crypt tight junctions as measured by freeze fracture techniques and, functionally less important, by an increase in the thickness of the subepithelial tissue layers in histological sections.

The first finding, that epithelial resistance only amounts to $20 \%$ of the total wall resistance, is of fundamental methodological relevance in the exploration of adaptive changes which are associated with structural alterations. Thus, for comparing the function of mucosae at different stages and patterns of intestinal adaptation, it is necessary to apply techniques which are able to discriminate between epithelial and subepithelial resistance.

The second finding is an example for the importance of the selective measurement of epithelial and subepithelial resistance which both are increased in the blind loop, but to different degrees: epithelial resistance increased three-fold as compared with the twofold subepithelial increase. From these functional data a morphological response in the blind loops could already be predicted.

The jejunum may be classified as one of the "very leaky' epithelia. As argued above, the conductivity of such epithelia is predominantly determined by the tight junctions. Thus, the increase in epithelial resistance points to alterations in this part of the epithelium. Therefore, freeze fracture electron microscopy on villus and crypt tight junctions has been performed. As a result of this study, the third essential finding was the considerable increase in the 'depth' (see Methods) of the tight junctions in the crypts. The number of 'strands' (see Methods), however, did not change thereby. Comparable tight junction alterations were seen in the gall bladder of Necturus maculosus under the influence of the plant cytokinin kinetin. ${ }^{9}$ Again, this was accompanied by an increase in epithelial resistance. In addition, subepithelial resistance increased also in the blind loop, the anatomical correlate of which was an increase in the thickness of the muscularis propria in the blind loop. This could be a consequence of the stasis of ingesta in the blind loop with concomitant work hypertrophy of the muscle layers.

Resistance values are usually related to the chamber opening area and thus to the serosal surface area of the intestine and do not take into account mucosal surface area alterations due to villus and/or crypt amplification. Thus, the epithelial resistance depends not only on the structure of the tight junctions, but also on the mucosal surface area of the intestine. A decrease in mucosal surface area could explain the increase in epithelial resistance observed in the blind loop. A morphometrical analysis however (on tissues fixed in the Ussing-chamber to guarantee an identical degree of stretch), revealed an increase in crypt surface area by $100 \%(\mathrm{p}<0.05)$ and in villus surface area by $25 \%$ (NS) of the blind loop mucosa as compared to control jejunum (unpublished data) ruling out that the increase in epithelial resistance was due to alterations in the mucosal surface area. Therefore, we attribute the increase in epithelial resistance to functional alterations of the tight junctions and conclude that the increase in tight junction 'depth' in the crypts indicates that such alterations have indeed taken place.

Sealing of the paracellular pathway by tight junction assembly is an active cell process. ${ }^{11}$ It supports absorption by reducing backleakage of absorbed ions through the tight junctions into the intestinal lumen. A similar teleological argument has been raised for the observed increase in gall bladder tight junction resistance after inhibition of active transport both by ouabain or by a Na-free solution. ${ }^{12}$ It is furthermore supported by recent findings in which an increased tight junction resistance in the gall bladder induced by protamine led to an increase in net water absorption. ${ }^{6}$ Thus, the increase in epithelial resistance in the blind loop represents an active adaptive process of the mucosa most likely to counterbalance cellular electrolyte malabsorption.

\section{References}

1 Riecken EO, Menge H. Nutritive effects of food constituents on the structure and function of the intestine. Acta Hepatogastroenterol 1977; 24: 389-99.

2 Bloch R, Menge H, Lorenz-Mayer H, Stöckert HG, Riecken EO. Functional, biochemical and morphological alterations in the intestines of rats with an experimental blind loop syndrome. Res Exp Med 1975; 166: 67-78.

3 Menge H, Köhn R, Dietermann KH, Lorenz-Mayer H, Riecken EO, Robinson JWL. Structural and functional alterations in the mucosa of self-filling blind loops in rats. Clin Sci 1979; 56: 121-31.

4 Gracey M. The contaminated small bowel syndrome: pathogenesis, diagnosis and treatment. Am J Clin Nutr 1979; 32: 234-43.

5 Schultz SG, Frizzell RA, Nellans HN. Ion transport by mammalian small intestine. Annu Rev Physiol 1974; 36: 51-91.

6 Fromm M, Palant CE, Bentzel CJ, Hegel U. Protamine reversibly decreases paracellular cation permeability in Necturus gallbladder. J Membr Biol 1985; 87: 141-50.

7 Fromm M, Schulzke J-D, Hegel U. Epithelial and subepithelial contributions to transmural electrical resistance of intact rat jejunum, in vitro. Pflügers Arch 1985; 405: 400-2.

8 Fromm M, Weskamp P, Hegel U. Versatile piezoelectric driver for cell puncture. Pflügers Arch 1980; 384: 6973.

9 Bentzel CJ, Hainau B, Ho S, et al. Cytoplasmic regulation of tight junction permeability: effect of plant cytokinins. Am J Physiol 1980; 239: C75-89. 
10 Kottra G, Frömter E. Rapid determination of intraepithelial resistance barriers by alternating current spectroscopy. II. Test of model circuits and quantification of results. Pflügers Arch 1984; 402: 421-32.

11 Griepp EB, Dolan WJ, Robbins ES, Sabatini DD. Participation of plasma membrane proteins in the formation of tight junctions by cultured epithelial cells. J Cell Biol 1983; 96: 693-703.

12 Bentzel CJ, Hainau BO. Cellular regulation of tight junction permeability in a resorptive epithelium. In: Mechanisms of intestinal secretion. New York: Liss Inc, 1979: 275-86. 